\title{
Radio emission altitude in pulsars
}

\author{
J. Kijak and J. Gil
}

\author{
Institute of Astronomy, University of Zielona Góra, Lubuska 2, 65-265 Zielona Góra, Poland \\ e-mail: jkijak@astro.ia.uz.zgora.pl; jag@astro.ia.uz.zgora.pl
}

Received 2 August 2002 / Accepted 22 October 2002

\begin{abstract}
The method of emission altitude estimation in radio pulsars based on pulse width measurements at the low intensity profile wings is reviewed. Both measurement details and model assumptions are discussed. It is argued that the pulse width measurement technique is sensitive enough to detect the radio emission generated at the beam edge near the last open field lines. We give the refined version of the formula for the radio emission altitudes and compare it with values obtained independently using a method based on measurements of separation between peaks of the outermost profile components. We show that both methods give comparable results.
\end{abstract}

Key words. stars: pulsars: general

\section{Introduction}

Thirty five years after the discovery of pulsars, the mechanism of their coherent radio emission is still unknown. Even the altitude of the emission region is still subject to some debate, although it is generally accepted that the radio emission originates within $10 \%$ of the light-cylinder radius $R_{\mathrm{LC}}=c P / 2 \pi$. It is obvious that all observational limits for emission altitude can be crucial for understading the physical mechanism of the observed coherent pulsar radio emission. One of the ways to estimate the emission altitude (Cordes 1978, 1993) involves an analysis of pulse width measurements corresponding to the low intensity profile wings (Fig. 1). This method was adopted and developed by Kijak \& Gil (1997, 1998, hereafter Papers I and II) and Kijak (2001, hereafter Paper III), using precise measurements of pulse profile width for a large number of pulsars. Three straightforward assumptions were used: (1) the pulsar radiation is narrow-band, with a radius-to-frequency mapping operating in the emission region (that is, a narrow-band of radio frequencies $\Delta v \ll \Delta v_{\text {tot }}$ is emitted at a given altitude $r_{\mathrm{em}}$, where $\Delta v_{\text {tot }} \sim 30 \mathrm{GHz}$ is the total pulsar band-width), (2) the elementary pulsar emission is relativistically beamed tangentially to dipolar magnetic field lines (beaming angle $\theta \sim 1 / \gamma$, where $\gamma \sim 100$ ), (3) the extreme profile wings originate at, or near the last open dipolar field lines. Therefore, the estimation of emission altitude is correct as long as the above assumptions are satisfied. In other words, if one can question one of these assumptions, our estimation of emission altitude can be questioned too. The first two assumptions are in agreement with basic pulsar electrodynamics (Goldreich \& Julian 1969; Sturrock 1971; Ruderman \& Sutherland 1975) and with the observed narrowing of profiles with increasing frequency

Send offprint requests to: J. Kijak,

e-mail: jkijak@astro.ia.uz.zgora.pl
(Thorsett 1991; Mitra \& Rankin 2002, hereafter MR). These two assumptions are commonly accepted. In the third assumption, the edge of pulsar radio beam is determined by the boundary of the GJ polar cap (Goldreich \& Julian 1969). This assumption is not widely accepted, and we give in this paper new arguments concerning its validity.

\section{Precise measurements of pulse profile and the edge of the radiation beam}

The important question is what we mean by the edge of pulsar beam and by the edge of a mean profile, respectively. The edge of pulsar beam is related to the edge of a profile in the following term: when the rotating pulsar beam starts passing through the radiotelescope, then the receiver starts detecting a weak coherent radio signal. The moment at which the pulsar beam enters the radiotelescope corresponds to the moment at which the recorded noise rapidly becomes a signal (and the signal becomes a noise when the beam leaves the radiotelescope). These moments are clearly visible in our log-scale profiles presented in polar coordinates (e.g. Fig. 1 in this paper and Fig. 2 in Paper I), and the corresponding longitudinal phases can be estimated within an accuracy of one resolution sample from these profiles. The edge of the beam is defined as the longitude at which recorded signals can be clearly distinguished from the noise and have the intensity at least $0.1 \%$ of the maximum intensity of the average profile.

Since the pulsar radiation is relativistically beamed along dipolar field lines, we can attempt to calculate which field lines should be tagged as coming from the emission region. This should be easiest for the last open magnetic field lines, which are believed to be associated with the lowest detectable level of radio emission at the profile wings. 


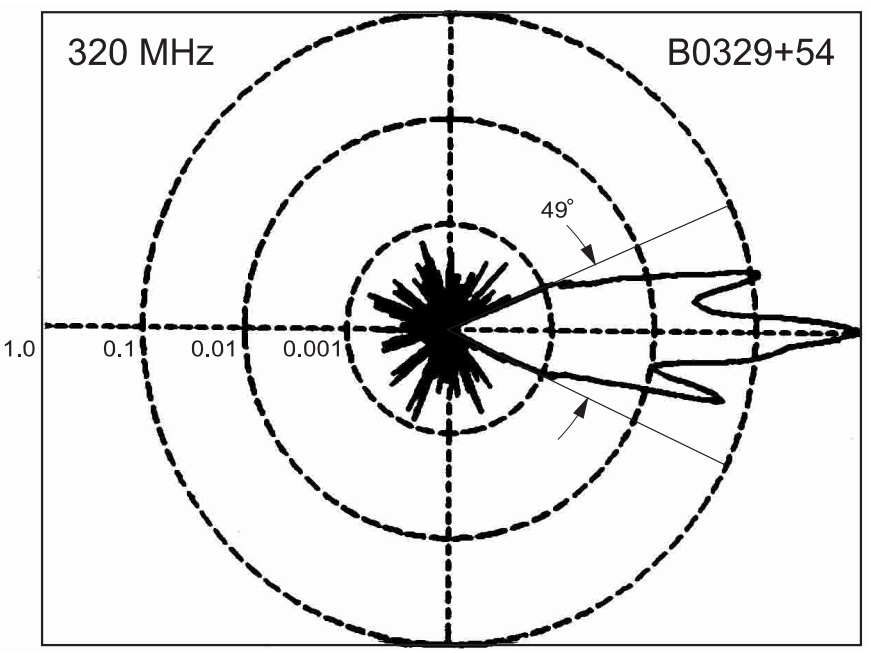

Fig. 1. A pulsar profile of $\mathrm{B} 0329+54$ at $320 \mathrm{MHz}$ in the log-scale and the polar coordinates (Hankins \& Fowler 1986). The data come from Jodrell Bank Observatory (Gould \& Lyne 1998).

The radio emission from pulsars is characterized by a short time of duration, typically $3-10 \%$ of its period. However, a few objects demonstrate the emission throughout the entire pulsar period. Hankins et al. (1993) showed, using the VLA, that the emission throughout the entire pulsar period is observed only in cases when the inclination angle $\alpha$ is smaller than the beam radius $\rho$, that is, the line-of-sight is always inside the emission beam (Gil 1985). In general, there is a rapid transition from the noise to the weak emission at the extreme profile wings (Fig. 1).

In our analysis of emission altitudes (Papers I, II and III), we measured pulse widths at the extremely low intensity level corresponding to about 0.05 per cent of the maximum intensity (see Fig. 1), using the polar-log-scale technique (Hankins \& Fowler 1986).

However, it is not certain if the entire GJ polar cap is involved in the emission process, that is, that the edge of pulsar beam is emitted at, or near the last open field lines corresponding to $s=1$ (where $s=d / r_{\mathrm{p}}$ is the mapping parameter describing the locus of corresponding field lines on the polar cap, $r_{\mathrm{p}}$ is the radius of the polar cap; see Eq. (2) in Paper I for details). It is possible that a forbidden area exists at the outer parts of the GJ polar cap. Evidently, we cannot give a theoretical answer to these questions. Recently, Gangadhara \& Gupta (2001) found new outermost weak components in the pulse window of pulsar B0329+54 at frequencies of 320 and $610 \mathrm{MHz}$. The separation $\Delta \phi$ between the peaks of newly detected components is $34^{\circ}$. In Fig. 1 we present the profile of PSR B $0329+54$ at $320 \mathrm{MHz}$, which was used by Kijak \& Gil (1998) in their measurement of pulse width and corresponding estimation of emission altitude (Paper II; Fig. 2). Let us note that the pulse width $W$ at $0.05 \%$ intensity level is about $49^{\circ}$, as compared with $34^{\circ}$ of peak separation reported by Gangadhara \& Gupta (2001). A comparison of these two measurements $\left(\Delta \phi=34^{\circ}\right.$ and $\left.W=49^{\circ}\right)$ clearly shows that the profile width presented in Fig. 1 includes new components found by Gangadhara \& Gupta (2001). Thus, the reported new outermost components in the profile of PSR B0329+54 do not contradict our assumption that $s \approx 1$ at the

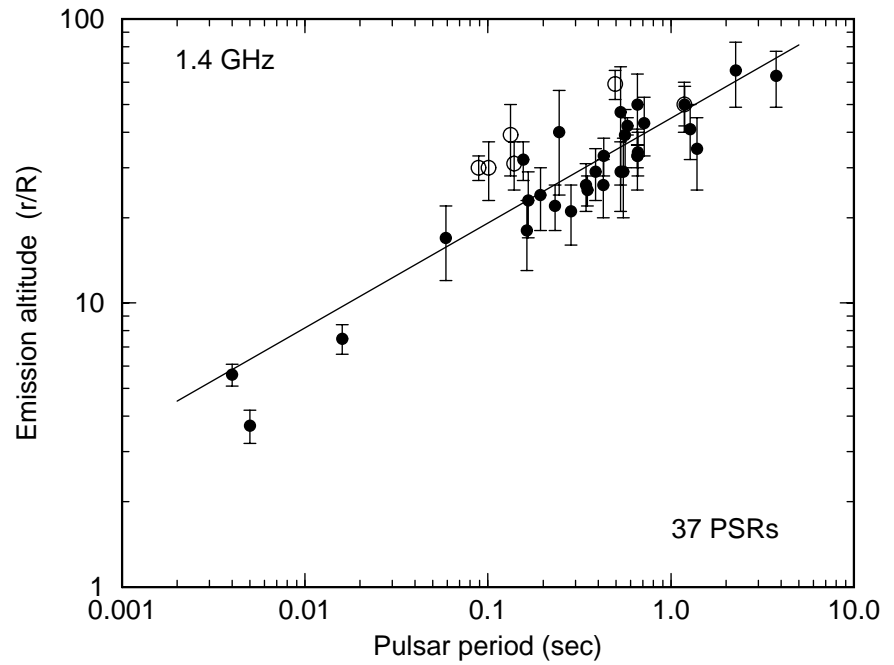

Fig. 2. Emission altitude at $1.4 \mathrm{GHz}$ versus pulsar period. Open circles correspond to young objects (see Paper III for details).

pulse edges. Our method of profile width measurements has proven to be sensitive to very weak radio emission at profile wings originating at the edge of the beam, most probably close to the last open field lines. The new outermost components detected by Gangadhara \& Gupta (2001) are clearly visible in Fig. 1 at the level of about $0.1 \%$ of the maximum intensity. Thus, high quality average profiles with the signal-to-noise ratio $S / N \sim 1000$ are needed to reveal low emission originating near the boudary of the last open field lines.

\section{Emission altitude estimation}

The result of emission altitude analysis (Papers I, II and III) indicated clearly that the emission altitude $r_{\mathrm{em}}$ depends not only on the observing frequency $v$, but also on the basic pulsar parameters: pulsar period $P$ and period derivative $\dot{P}$. In order to verify ultimately the emission altitude estimation given in previous papers, we present in this paper the analysis of the largest available data set (37 PSRs) obtained at $1.4 \mathrm{GHz}$ in Effelsberg Observatory between 1995 and 1998. An apparent period dependence of emission altitudes is clearly visible in Fig. 2, where a formal fit to all data points gives $r_{\mathrm{em}}=$ $(45 \pm 3) R P^{0.37 \pm 0.05}$. This result confirms the previous analysis of the emission altitude for a smaller data set (Paper I).

Figure 2 also shows a weak dependence of $r_{\mathrm{em}}$ on pulsar characteristic age $\tau_{6}$ (in $10^{6} \mathrm{yrs}$ ), which is discussed in Paper III (Fig. 2 and Table 2). A formal fit to the same data set (37 PSRs) at $1.4 \mathrm{GHz}$ yields $r_{\mathrm{em}}=(33 \pm 1) R \tau_{6}^{-0.07 \pm 0.03}$, confirming the previous result. The emission altitudes differ slightly between very young and very old pulsars with approximately the same period.

In Paper II, the radius-to-frequency mapping was derived for 16 typical pulsars covering the frequency range between 0.3 and $20 \mathrm{GHz}$. The average frequency dependence on emission altitude is represented by the formal fit $r_{\mathrm{em}} \propto v^{-0.26 \pm 0.09}$. We also studied three millisecond pulsars in the frequency range between 0.3 and $5 \mathrm{GHz}$, which seem to behave similarly to the typical pulsars. 

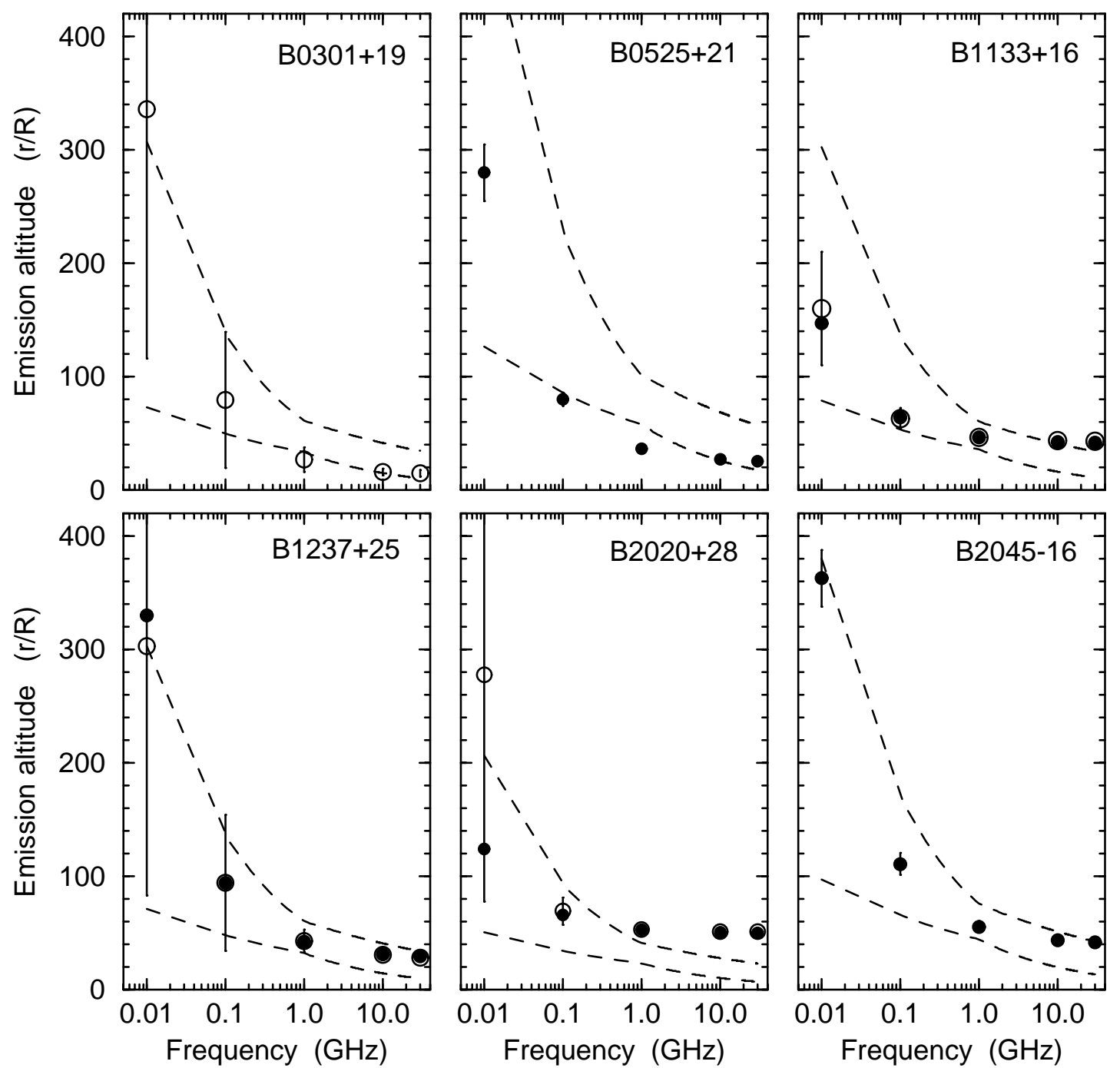

Fig. 3. The multifrequency study of emission altitude (see also Kijak 2002). Data points represent calculations and their errors from the paper of Mitra \& Rankin (2002). The dashed lines show upper and lower limits, corresponding to limiting values of parameters in Eq. (2).

The radial location of the emission regions was discussed in Papers I, II and III. A general form of the expression for the emission altitude is

$r_{\mathrm{KG}}=\mathcal{A} R v_{\mathrm{GHz}}^{-a} \tau_{6}^{-b} P^{c}$,

where $R \cong 10^{6} \mathrm{~cm}$ is the neutron star radius, $v_{\mathrm{GHz}}$ is the observing frequency (in GHz), $\tau_{6}$ is the characteristic age (in $10^{6} \mathrm{yrs}$ ) and $P$ is the pulsar period (in s). Taking into account new results presented above, we obtain the verified formula

$r_{\mathrm{KG}}=(50 \pm 10) R v_{\mathrm{GHz}}^{-0.26 \pm 0.09} \tau_{6}^{-0.07 \pm 0.03} P^{0.37 \pm 0.05}$.

Using $\tau_{6}=16 P / \dot{P}_{-15}$ and $\dot{P}_{-15}=\dot{P} / 10^{15}$ we obtain an alternative form of Eq. (2)

$r_{\mathrm{KG}} \approx(40 \pm 8) R v_{\mathrm{GHz}}^{-0.26 \pm 0.09} \dot{P}_{-15}^{0.07 \pm 0.03} P^{0.30 \pm 0.05}$,

where $\dot{P}$ is the period derivative. The uncertainties given for various quantities in the above equations follow from systematic errors in the pulse width $W$ measurements and random errors in estimations of the inclination angle $\alpha$ between the rotation and magnetic axes and the impact angle $\beta$ of the closest approach of the observer to the magnetic axis (see Papers I and II). There is a number of methods of estimation of $\alpha$ and $\beta$ angles. Although they use slightly different assumptions (Lyne \& Manchester 1988; Rankin 1990; Blaskiewicz et al. 1991; Gould 1994), it is critical for all of them that good polarization data are used. The uncertainty in the polarization position angle (hereafter PA) is due to random noise, erros in the position of the antenna feed, uncertainties in the observing centre frequency, and errors in estimating the ionospheric Faraday rotation (e.g. van Ommen et al. 1997). The estimated uncertainties in $\alpha$ and $\beta$ angles follow from errors in PA determination and longitudinal phase resolution (e.g. Miller \& Hamilton 1993). Accurate polarization measurements can yield reasonable estimates of $\alpha$ and $\beta$ angles. In our analysis, we used high quality pulsar profiles for which good polarization measurements were available. Moreover, we only selected those pulsars in which different methods mentioned above gave converging results (see Table 1 in Paper I). Typical uncertainties of $\alpha$ and $\beta$ estimates are $\$ 30 \%$ (Blaskiewicz et al. 1991; van Ommen et al. 1997; Manchester et al. 1998, Paper I), while uncertainties of pulse width $W$ measurements are $\lesssim 15 \%$ (see Papers I, II and III). It is important for our method that an apparent pulsar 
beam spreads out about equally at both sides of the so-called fiducial plane, containing both the rotation and the magnetic axes (although no symmetry in the observed intensities is required). Therefore, we excluded a number of pulsars with obviously missing components (Papers I and II), which can be judged using the symmetry of the polarization position angle swing and/or distribution of circular polarization.

\section{Discussion and conclusions}

As it follows from Eqs. (2) or (3), the radio emission of pulsars originates at the narrow altitude range below 10 per cent of the light-cylinder radius. In millisecond pulsars, the radio emission region is much more compact, consistent with their small lightcylinder radii, and is located closer to the neutron star surface (see Paper II). Previously, it was thought that millisecond pulsars have different characteristics of radio emission from those of normal pulsars. However, it was recently argued (Kramer et al. 1999; Gil \& Sendyk 2000) that millisecond pulsar emission properties do not differ from those of typical pulsars.

Gil et al. (2002) analysed simultaneous dual-frequency single pulse observations of PSR B0329+54 at 240 and $610 \mathrm{MHz}$. The phase shifts of the leading and the trailing conal components are not equal at these frequencies. This is caused by the retardation-aberration effects. In fact, since the conal beams at different frequenies are emitted at different altitudes, lower frequency radiation arrives earlier. The retardation-aberration shift of $0.39^{\circ}$ translates into a difference of emission altitudes of $\Delta r \sim 2.3 \times 10^{7} \mathrm{~cm}$. This is consistent with the result obtained using the semi-empirical formula $\Delta r_{\mathrm{KG}}=2 \times 10^{7} \mathrm{~cm}$ (Eqs. (2) or (3)).

Mitra \& Rankin (2002) found the radius-to-frequency mapping for a number of pulsars using different method based on measurements of separation between the outer-conal components. In Fig. 3 we present a comparison between their results and those following from Eqs. (2) or (3). Emission altitudes represented by open and filled circles were calculated from $r_{\mathrm{MR}}=h \cdot s^{-2}$, where $h$ was the emission height obtained by Mitra \& Rankin (2002) for conal component, and the parameter $s$ was taken as the mean value for each pulsar, respectively. The open circles represent calculations for the first group of their fitting procedure, and filled circles were calculated using the index of $-2 / 3$ (see Mitra \& Rankin 2002, their Table 4). Figure 3 shows that our semi-empirical formula (Eq. (2)) describes the radio emission altitudes $r_{\mathrm{KG}}$ quite well, in consistency with independently obtained values $r_{\mathrm{MR}}$.

Acknowledgements. This work was in part supported by the Polish State Committe for Scientific Research Grant 2 P03D 008 19. We thank Prof. Dr. R. Wielebinski for hospitality at the MPIfR in Bonn, where a part of this work was done. Moreover authors would like to thank the referee, Dr. A. Harding, for usful advice. We also thank U. Maciejewska for technical help.

\section{References}

Blaskiweicz, M., Cordes, J. M., \& Wasserman, I. 1991, ApJ, 370, 643 Cordes, J. M. 1978, ApJ, 222, 1006

Cordes, J. M. 1993, in Proc. Los Alamos Workshop, ed. K. A. van Riper, R. Epstein, \& C. Ho (Cambridge Univ. Press, Cambridge), 182

Gangadhara, R. T., \& Gupta, Y. 2001, ApJ, 555, 31

Gil, J., Gronkowski, P., \& Rudnicki, W. 1984, A\&A, 132, 312

Gil, J. 1985, ApJ, 299, 154

Gil, J., \& Sendyk, M. 2000, ApJ, 541, 351

Gil, J., Gupta, Y., Gothoskar, P. B., \& Kijak, J. 2002, ApJ, 565, 500

Goldreich, P., \& Julian, W. H. 1969, ApJ, 157, 869

Gould, M. 1994, Ph.D. Thesis, Univ. of Manchester

Gould, M., \& Lyne, A. G. 1998, MNRAS, 301, 235

Hankins, T. H., \& Fowler, L. A. 1986, ApJ, 304, 256

Hankins, T. H., Moffett, D. A., Novikow, A., \& Popov, M. 1993, ApJ, 417,735

Kijak, J., \& Gil, J. 1997, MNRAS, 288, 631 (Paper I)

Kijak, J., \& Gil, J. 1998, MNRAS, 299, 855 (Paper II)

Kijak, J. 2001, MNRAS, 323, 537 (Paper III)

Kijak, J. 2002, in Proc. of the 270. WE-Heraeus Seminar, ed. W. Becker, H. Lesch, \& J. Trümper, MPE-Report 278, 200

Kramer, M., Lange, C., Lorimer, D. R., et al. 1999, ApJ, 526, 957

Lyne, A. G., \& Manchester, R. N. 1988, MNRAS, 234, 477

Manchester, R. N., Han, J. L., \& Qiao, G. J. 1998, MNRAS, 295, 280

Miller, M. C., \& Hamilton, R. J. 1993, ApJ, 411, 298

Mitra, D., \& Rankin, J. M. 2002, ApJ, 577, 322

Rankin, J. M. 1990, ApJ, 352, 247

Ruderman, M. A., \& Sutherland, P. G. 1975, ApJ, 196, 51

Sturrock, P. A. 1971, ApJ, 164, 529

Thorsett, S. E. 1991, ApJ, 377, 263

Xilouris K. M. 1997, A\&A, 324, 981

van Ommen, T. D., D’Alessandro, R., Hamilton, P. A., \& McCulloch, P. M. 1997, MNRAS, 287, 307 